\title{
New Cosmic Microwave Background Constraint to Primordial Gravitational Waves
}

\author{
Tristan L. Smith, Elena Pierpaoli, and Marc Kamionkowski \\ California Institute of Technology, Mail Code 130-33, Pasadena, California 91125, USA
}

(Received 6 March 2006; revised manuscript received 26 May 2006; published 14 July 2006)

\begin{abstract}
Primordial gravitational waves (GWs) with frequencies $\gtrsim 10^{-15} \mathrm{~Hz}$ contribute to the radiation density of the Universe at the time of decoupling of the cosmic microwave background (CMB). This affects the $\mathrm{CMB}$ and matter power spectra in a manner identical to massless neutrinos, unless the initial density perturbation for the GWs is nonadiabatic, as may occur if such GWs are produced during inflation or some post-inflation phase transition. In either case, current observations provide a constraint to the GW amplitude that competes with that from big-bang nucleosynthesis (BBN), although it extends to much lower frequencies ( $\sim 10^{-15} \mathrm{~Hz}$ rather than the $\sim 10^{-10} \mathrm{~Hz}$ from BBN): at $95 \%$ confidence level, $\Omega_{\mathrm{gw}} h^{2} \lessgtr$ $8.4 \times 10^{-6}$ for homogeneous (i.e., nonadiabatic) initial conditions. Future CMB experiments, like Planck and CMBPol, should allow sensitivities to $\Omega_{\mathrm{gw}} h^{2} \lesssim 1.4 \times 10^{-6}$ and $\Omega_{\mathrm{gw}} h^{2} \lesssim 5 \times 10^{-7}$, respectively.

DOI: 10.1103/PhysRevLett.97.021301

PACS numbers: 98.80.Es, 95.85.Sz, 98.70.Vc
\end{abstract}

There are many conjectured sources of a primordial cosmological gravitational-wave background (CGWB), including inflation, pre-big-bang theories, phase transitions, or the ekpyrotic model [1]. Such backgrounds are among the targets of the Laser Interferometric Gravitational-Wave Observatory (LIGO), and they will be sought with future observatories, such as NASA's Laser Interferometer Space Antenna (LISA), the Big-Bang Observer (BBO), and Japan's Deci-Hertz Interferometer Gravitational-wave Observatory (DECIGO).

The CGWB amplitude is constrained at the lowest observable frequencies, $\sim 10^{-17}-10^{-16} \mathrm{~Hz}$ (corresponding to wavelengths comparable to the cosmological horizon today), by large-angle fluctuations in the cosmic microwave background (CMB) temperature [2]. Prospects for probing lower CGWB amplitudes at these frequencies come from future measurements of the polarization of the $\mathrm{CMB}[3,4]$. Apart from a window around $10^{-9}-10^{-8} \mathrm{~Hz}$, where the CGWB is constrained by pulsar timing [5,6], the strongest constraint to the CGWB amplitude for frequencies greater than $\sim 10^{-10} \mathrm{~Hz}$ comes from big-bang nucleosynthesis (BBN) [7]. The lower limit to the frequency range is determined by the comoving horizon size at the time of BBN. Primordial gravitational waves of shorter wavelengths, or larger frequencies, contribute to the radiation density at the time of BBN, thereby increasing the expansion rate and thus the light-element abundances. Measurements of light-element abundances limit the number of additional relativistic species at BBN to the equivalent of 1.4 neutrino degrees of freedom [8], which translates to a limit to a current CGWB energy density $\Omega_{\mathrm{gw}} h^{2} \leqslant 7.8 \times$ $10^{-6}$.

The frequency range $\sim 10^{-16}-10^{-10} \mathrm{~Hz}$ remains largely unconstrained. An upper limit $\Omega_{\mathrm{gw}} h^{2} \lesssim 0.1$ can be placed in this frequency range from quasistellar object (QSO) astrometry $[9,10]$. It has been proposed that future measurements of anisotropy in the global rate of change of observed redshifts might someday get down to $\Omega_{\mathrm{gw}} h^{2} \sim$ $10^{-5}[11]$.
Here we note that recent measurements of the angular power spectrum of the $\mathrm{CMB}$ that constrain the nonrelativistic-matter density $\Omega_{m} h^{2}$ to roughly $10 \%$ [12] are, to a first approximation, constraints to the radiation energy density at the time of CMB decoupling; the constraint corresponds to a limit of a few extra neutrino degrees of freedom. From this, we infer that the CMB provides a limit to $\Omega_{\mathrm{gw}} h^{2}$ that may be competitive with that from BBN, but extends to the lower frequencies, $\sim 10^{-15} \mathrm{~Hz}$, corresponding to wavelengths comparable to the comoving horizon at CMB decoupling [13]. This limit therefore improves upon previous constraints over the frequency interval $10^{-15}-10^{-10} \mathrm{~Hz}$ by 4 orders of magnitude.

More precisely, the CGWB behaves as a free-streaming gas of massless particles, just like massless neutrinos, and therefore affects the growth of density perturbations in ways in addition to its effect on the expansion rate at decoupling. If the CGWB energy-density perturbations are adiabatic (i.e., have the same density distribution as other relativistic species), then the effects of the CGWB on the CMB/large-scale structure (LSS) are indistinguishable from those due to massless neutrinos. In this case, $\mathrm{CMB} /$ LSS constraints to the number of massless-neutrino species [14] translate directly to a constraint to the CGWB energy density. If, however, the primordial perturbations to the CGWB energy-density perturbations are nonadiabatic, as might be expected if they are produced by inflation, prebig-bang models, ekpyrotic, or phase transitions and/or cosmic turbulence [see, e.g., Ref. [15]], then the CMB/ LSS effects of the CGWB may differ from those of adiabatic massless neutrinos.

In this Letter, we carry out a detailed analysis of current constraints to the CGWB amplitude that come from current measurements of the $\mathrm{CMB}$ power spectrum and matter power spectrum. Our calculations of the CMB and matter power spectra include the effects of the CGWB on the expansion rate and on the growth of perturbations, for both adiabatic and nonadiabatic initial conditions for the 
CGWB. We include current constraints from the CMB, galaxy surveys, and the Lyman- $\alpha$ forest. We then forecast how these constraints may be improved with future CMB measurements.

We first consider the case when the CGWB has adiabatic initial conditions. In this case, the effects of the CGWB on the expansion history and structure formation are identical to those of massless neutrinos. The analysis proceeds just as in Ref. [14]. We have updated this analysis to include the third year WMAP data release, new small-scale CMB results, as well as constraints from the Lyman- $\alpha$ forest. The CMB results we use are from WMAP3, ACBAR, CBI, VSA, and BOOMERanG, and we use the measurement of the Galaxy power spectrum from the $2 \mathrm{dF}$ Galaxy Redshift Survey and the Sloan Digital Sky Survey (SDSS), and the Lyman- $\alpha$ forest [16]. We implement the Lyman- $\alpha$ constraints following the method discussed in Ref. [17], with minor modifications that were suggested by the authors. To translate the constraint to the number of extra neutrino degrees of freedom to a CGWB energy density, we use the relation $\Omega_{\mathrm{gw}} h^{2}=5.6 \times 10^{-6}$, the density contributed by a single massless-neutrino species for a monochromatic CGWB spectrum.

Results for adiabatic initial conditions are shown in Fig. 1. A limit at $95 \%$ C.L. of $\Omega_{\mathrm{gw}} h^{2} \leqslant 4.0 \times 10^{-5}$ is obtained from a combination of current CMB data, galaxy power spectrum, and the Lyman- $\alpha$ forest, and under the assumption that the number of neutrino degrees of freedom is $N_{\nu}=3.04$ and that neutrino masses are free to vary. Because of a slight discrepancy between the matter power spectrum from the best-fit CMB model and that measured in galaxy surveys and Lyman- $\alpha$ forest measurements, the addition of galaxy surveys and the Lyman- $\alpha$ forest weakens the bound by roughly a factor of 2 . A small CGWB component improves slightly the $\mathrm{CMB}+$ galaxy $+\mathrm{Ly} \alpha$ agreement (cf., the solid curve in Fig. 1), although the difference between $N_{\mathrm{gw}}=0$ and $N_{\mathrm{gw}}=2$ is statistically insignificant. Although not shown, we find that the exclusion of the Lyman- $\alpha$ forest weakens the CMB + galaxy + $\operatorname{Ly} \alpha$ bound only slightly. If neutrino masses are assumed to be undetermined, then the $\mathrm{CMB}+$ galaxy $+\mathrm{Ly} \alpha$ bound is shifted by approximately two neutrinos (cf., the dot-dashed curve in Fig. 1), which indicates that there is a degeneracy between the neutrino mass and the CGWB. This same trend has been observed in Ref. [18]. Note that the bound is improved by roughly a factor of 2.5 if we include only current CMB data.

If the CGWB is produced from quantum fluctuations to the spacetime metric during the same superluminal expansion that produced primordial density perturbations (e.g., from inflation, but also from pre-big-bang or ekpyrotic scenarios), then primordial perturbations to the CGWB density should be nonadiabatic. In such scenarios, the particle species in the primordial Universe are all produced by decay of the inflaton; this is why inflation produces

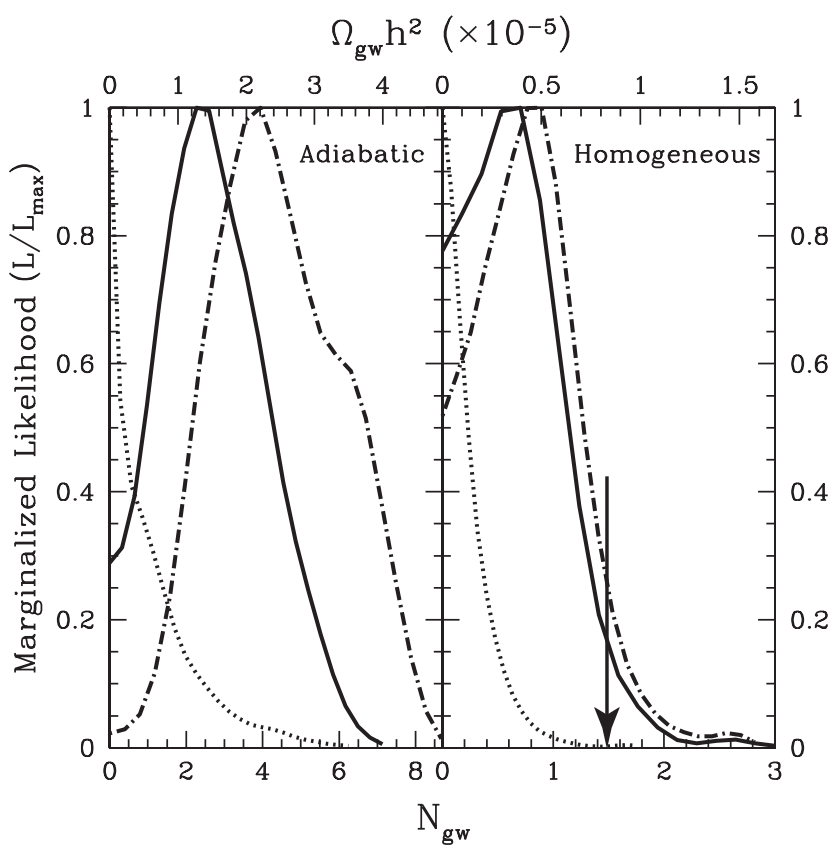

FIG. 1. Adiabatic: the marginalized (unnormalized) likelihoods for the CGWB energy density if perturbations to the CGWB density are adiabatic. The dotted curve is the result obtained using only CMB data. The thick solid curve includes galaxies as well as the Lyman- $\alpha$ forest. In all of the aforementioned curves, the marginalization is over the nonrelativisticmatter density $\Omega_{m} h^{2}$, baryon density $\Omega_{b} h^{2}$, scalar spectral index $n_{s}$, power-spectrum amplitude $A_{s}$, the optical depth $\tau$ to the surface of last scatter, and the angle $\theta$ subtended by the first acoustic peak (marginalization over $\theta$ essentially stands in for marginalization over the Hubble constant). We hold the geometry fixed to flat, the number of neutrinos to $N_{\nu}=3.04$, and the neutrino masses fixed to zero. Finally, the dot-dashed curve (to the right) shows current constraints from the $\mathrm{CMB}+$ galaxies + Ly $\alpha$ if we allow for and marginalize over nonzero neutrino masses as well. The number of equivalent neutrino degrees of freedom $\left(N_{\mathrm{gw}}\right)$ is shown on the bottom axis. Homogeneous: same as the left panel, except for homogeneous initial conditions for the CGWB. The arrow indicates the 95\% C.L. upper limit $\Omega_{\mathrm{gw}} h^{2} \leq 8.4 \times 10^{-6}$ that we adopt as our central result. This is obtained from the analysis that includes current $\mathrm{CMB}+$ galaxy $+\operatorname{Ly} \alpha+$ free $m_{\nu}$.

primordial adiabatic perturbations: i.e., the fractional perturbation to the energy density of all the particle species is the same. However, gravitational waves are produced during inflation by quantum fluctuations in tensor perturbations to the spacetime metric - not through decay of the inflaton. The CGWB should therefore not have the same primordial energy-density perturbations as the particle species; in fact, in linear theory, there should be no primordial perturbation to the CGWB amplitude. We therefore redo our likelihood analysis assuming the CGWB has homogeneous initial conditions. More precisely, we have chosen to set the initial CGWB density perturbation to zero in the conformal Newtonian gauge. With this ansatz, the 
primordial curvature perturbation vanishes in the limit that the CGWB energy density dominates, as it should; the curvature perturbation approaches the standard adiabatic perturbation in the limit that the CGWB vanishes, also as it should. In this limit (appropriate for our analysis), selfconsistency of the perturbation equations demands that nonzero higher-order moments in the CGWB distribution function are induced at early times.

If the CGWB is initially homogeneous, then the initial conditions for the CGWB perturbations differ from those for massless neutrinos. This affects the growth of perturbations, especially at large scales, and the degeneracy between the CGWB and massless neutrinos is thus broken. The bound to the CGWB then turns out to be stronger than in the adiabatic case. Figure 1 shows results for the likelihood for $\Omega_{\mathrm{gw}} h^{2}$ for different combinations of current data sets as well as forecasts for the likelihoods expected when future CMB experiments are included. If the CGWB is produced by some mechanism that leaves its primordial density uncorrelated with the curvature perturbatione.g., inflation or perhaps some post-inflation phasetransition mechanism - then this is the result that should be applicable. We adopt as our 95\% C.L. upper bound, $\Omega_{\mathrm{gw}} h^{2} \lesssim 8.4 \times 10^{-6}$, for homogeneous CGWB initial conditions from the combination of data from current $\mathrm{CMB}$ experiments, galaxy surveys, and the Lyman- $\alpha$ forest and under the assumption that the number of neutrino degrees of freedom is $N_{\nu}=3.04$ and that neutrino masses are free to vary. Note, again, that the bound would be roughly twice as strong if we were to restrict ourselves only to CMB data. And again, although not shown, we find that the exclusion of the Lyman- $\alpha$ forest weakens the $\mathrm{CMB}+$ galaxy $+\operatorname{Ly} \alpha$ bound only slightly.

Our central results are summarized in Fig. 2, which shows $\Omega_{\mathrm{gw}} h^{2}$ versus gravitational-wave frequency. Our new constraints are competitive with the BBN constraint over the frequency range where both constraints apply. The precise value of the $\mathrm{BBN}$ constraint depends on the precise constraint to the maximum number of neutrino degrees of freedom allowed by BBN. Some authors [19] claim a limit $\left(N_{\nu}-3.04\right) \lesssim 0.2$ (at $95 \%$ C.L.), but more recent and conservative estimates (that include new ${ }^{4} \mathrm{He}$ measurements and the CMB value for the baryon density) [8], which we choose to adopt, place the limit at $\left(N_{\nu}-3.04\right) \lesssim$ 1.4, comparable to the CMB/LSS bound we have derived. However, our new results apply four decades lower in frequency, and provide the strongest constraint to the CGWB amplitude over the frequency range $10^{-15}-10^{-10} \mathrm{~Hz}$.

To forecast the sensitivity of future $\mathrm{CMB}$ experiments to the CGWB, we have carried out a Fisher analysis that shows that when Planck and CMBPol fly, the sensitivity should be increased by a factor of roughly 10 , while the BBN constraint may continue to be limited by the same astrophysical systematic uncertainties. See Table I for the experimental specifications used in our Fisher analysis. In

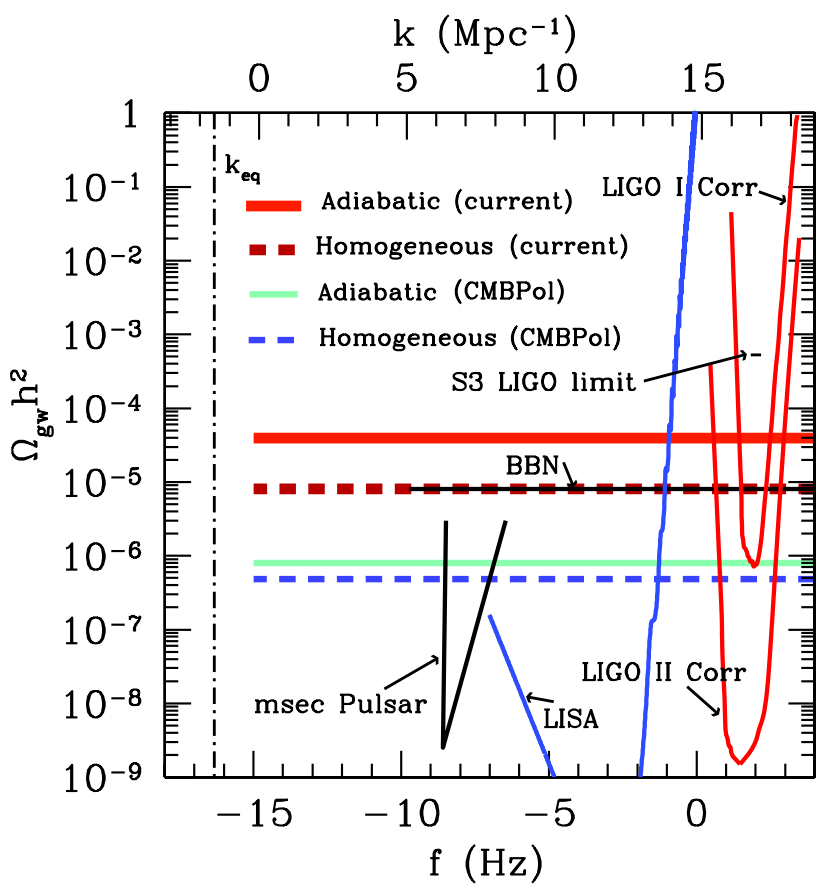

FIG. 2 (color online). The gravitational-wave density $\Omega_{\mathrm{gw}} h^{2}$ vs frequency. The BBN constraint corresponds to a limit of 1.4 extra neutrino degrees of freedom. We also show our constraints, from current CMB, galaxy, and Lyman- $\alpha$ data, for a CGWB with adiabatic primordial perturbations ["adiabatic (current)"] and for homogeneous initial conditions ["homogeneous (current)"], as well as our forecasts for the sensitivities if current $\mathrm{CMB}$ data are replaced by data from CMBPol. These constraints apply to a monochromatic CGWB spectrum. Also shown are the reaches of LIGO and LISA. BBO (not shown) should go deeper, but primarily at frequencies $\sim 1 \mathrm{~Hz}$. Large-angle CMB fluctuations (also not shown) constrain $\Omega_{\mathrm{gw}} h^{2} \lesssim 10^{-10}$ for a scaleinvariant spectrum at frequencies $\lesssim 10^{-17} \mathrm{~Hz}$. The LIGO S3 upper limit is from Ref. [21] and the msec pulsar curve is from Refs. [5,6].

our Fisher analysis we included the improved CMB observations as well as the current galaxy and Lyman- $\alpha$ constraints and allowed $m_{\nu}$ to vary with $N_{\nu}=3.04$.

We have not determined precisely the lower end of the frequency range for which our bound applies. In order for the constraint to apply, the gravitational-wave wavelength must be within the horizon at roughly the time of, or

TABLE I. CMB experimental specifications for Fisher matrix.

\begin{tabular}{lcrrrc}
\hline \hline Experiment & $\theta_{\text {beam }}$ & $\left(w_{T}\right)^{-1 / 2}$ & $\left(w_{P}\right)^{-1 / 2}$ & $f_{\text {sky }}$ & $\Omega_{\mathrm{gw}} h^{2}$ \\
\hline Planck: & 7.1 & 42.2 & 80.5 & 0.8 & $1.4 \times 10^{-6}$ \\
& 5.0 & 64.8 & 132.3 & $\ldots$ & $\ldots$ \\
CMBPol: & 4.0 & 1.0 & 1.4 & 0.8 & $5 \times 10^{-7}$ \\
\hline \hline
\end{tabular}

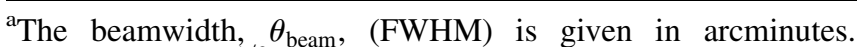
Weights, $\left(w_{T, P}\right)^{-1 / 2}$, are in arcminutes $\mu \mathrm{K}$. The sky fraction is given by $f_{\text {sky. }}$. The sensitivities to $\Omega_{\mathrm{gw}} h^{2}$ are $95 \%$ C.L. for homogeneous initial conditions. 
slightly before, recombination. Otherwise, the waves do not propagate as massless modes. Analytic and numerical integrations of the mode equations for gravitational waves in an expanding universe [e.g., Fig. 2 in Ref. [20] ], indicate that the mode is oscillating when $k \tau \simeq 10$, where $k$ is the wave number and $\tau$ the conformal time evaluated at decoupling. This translates to a frequency $\nu \simeq 5 \times 10^{-17} \mathrm{~Hz}$. More realistically, the gravitational wave will need to oscillate for a while before recombination in order to have the effects we have considered here. We therefore tentatively estimate $10^{-15} \mathrm{~Hz}$ as the lowest frequency for which our bound applies, although the precise value may differ slightly. We leave a more precise calculation for future work.

There is also a slight correction if our bound is applied to a scale-invariant spectrum. In this case, the number of gravitational-wave modes propagating as massless modes changes with time, as more modes enter the horizon. As a result, the energy density does not scale with scale factor $a$ simply as $a^{-4}$. This, however, produces only a logarithmic correction, which is within the theoretical error of the treatment we have presented here.

Finally, we point out that the limit is probably not relevant for scale-invariant spectra, such as those produced by inflation. Those are already constrained to be roughly 9 orders of magnitude lower in amplitude, at slightly lower frequencies $\sim 10^{-17} \mathrm{~Hz}$, from large-angle fluctuations in the CMB. However, phase transitions or other exotic mechanisms that produce a CGWB at frequencies $\geq 10^{-15} \mathrm{~Hz}$ will now face this new constraint.

T.L.S. was supported by the NSF. E.P. was supported by NSF Grant No. AST-0340648, also supported by NASA Grant No. NAG5-11489. This work was supported in part by DOE Grant No. DE-FG03-92-ER40701 and NASA Grant No. NNG05GF69G. This work was supported in part by the NSF through the TeraGrid resources provided by NCSA and SDSC under TeraGrid Grant No. AST050005T.

[1] M. Maggiore, Phys. Rep. 331, 283 (2000).

[2] L.F. Abbott and M. B. Wise, Nucl. Phys. B 244, 541 (1984); A. A. Starobinsky, Sov. Astron. Lett. 11, 133 (1985); V. A. Rubakov, M. V. Sazhin, and A. V. Veryaskin, Phys. Lett. 115B, 189 (1982); R. Fabbri and M. D. Pollock, Phys. Lett. 125B, 445 (1983).

[3] M. Kamionkowski, A. Kosowsky, and A. Stebbins, Phys. Rev. Lett. 78, 2058 (1997).

[4] U. Seljak and M. Zaldarriaga, Phys. Rev. Lett. 78, 2054 (1997).

[5] A. N. Lommen, astro-ph/0208572.

[6] V. M. Kaspi, J. H. Taylor, and M.F. Ryba, Astrophys. J. 428, 713 (1994).
[7] B. Allen, gr-qc/9604033.

[8] R. H. Cyburt, B. D. Fields, K. A. Olive, and E. Skillman, Astroparticle Physics 23, 313 (2005).

[9] T. Pyne, C. R. Gwinn, M. Birkinshaw, T. M. Eubanks, and D. N. Matsakis, Astrophys. J. 465, 566 (1996).

[10] C. R. Gwinn, T. M. Eubanks, T. Pyne, M. Birkinshaw, and D. N. Matsakis, Astrophys. J. 485, 87 (1997).

[11] N. Seto and A. Cooray, Phys. Rev. D 73, 023005 (2006).

[12] M. Kamionkowski and A. Kosowsky, Ann. Rev. Nucl. Part. Sci. 49, 77 (1999); P. de Bernardis et al. (BOOMERanG Collaboration), Nature (London) 404, 955 (2000); A. D. Miller et al., Astrophys. J. 524, L1 (1999); S. Hannay et al., Astrophys. J. 545, L5 (2000); N. W. Halverson et al., Astrophys. J. 568, 38 (2002); B. S. Mason et al., Astrophys. J. 591, 540 (2003); A. Benoit et al. (Archeops Collaboration), Astron. Astrophys. 399, L25 (2003); J. H. Goldstein et al., Astrophys. J. 599, 773 (2003); D. N. Spergel et al. (WMAP Collaboration), Astrophys. J. Suppl. Ser. 148, 175 (2003).

[13] This estimate was provided in Fig. 2 of Ref. [22].

[14] E. Pierpaoli, Mon. Not. R. Astron. Soc. 342, L63 (2003).

[15] M.S. Turner and F. Wilczek, Phys. Rev. Lett. 65, 3080 (1990); A. Kosowsky and M. S. Turner, Phys. Rev. D 47, 4372 (1993); M. Kamionkowski, A. Kosowsky, and M. S. Turner, Phys. Rev. D 49, 2837 (1994); A. Kosowsky, A. Mack, and T. Kahniashvili, Phys. Rev. D 66, 024030 (2002); A. D. Dolgov, D. Grasso, and A. Nicolis, Phys. Rev. D 66, 103505 (2002); L. A. Boyle, P. J. Steinhardt, and N. Turok, Phys. Rev. D 69, 127302 (2004).

[16] G. Hinshaw et al. (WMAP Collaboration), astro-ph/ 0603451; L. Page et al. (WMAP Collaboration), astroph/0603450; C.-1. Kuo et al. (ACBAR Collaboration), Astrophys. J. 600, 32 (2004); A. C.S. Readhead et al., Astrophys. J. 609, 498 (2004); C. Dickinson et al., Mon. Not. R. Astron. Soc. 353, 732 (2004); W. C. Jones et al., astro-ph/0507494; F. Piacentini et al., astro-ph/0507507; T. E. Montroy et al., astro-ph/0507514; M. Colless et al. (The 2dFGRS Collaboration), Mon. Not. R. Astron. Soc. 328, 1039 (2001); W.J. Percival et al. (The 2dFGRS Collaboration), Mon. Not. R. Astron. Soc. 327, 1297 (2001); M. Tegmark et al. (The SDSS Colalboration), Astrophys. J. 606, 702 (2004); M. Viel, M. G. Haehnelt, and V. Springel, Mon. Not. R. Astron. Soc. 354, 684 (2004).

[17] M. Viel, J. Weller, and M. Haehnelt, Mon. Not. R. Astron. Soc. 355, L23 (2004).

[18] P. Crotty, J. Lesgourgues, and S. Pastor, Phys. Rev. D 69, 123007 (2004).

[19] S. Burles, K. M. Nollett, J. W. Truran, and M. S. Turner, Phys. Rev. Lett. 82, 4176 (1999).

[20] J. R. Pritchard and M. Kamionkowski, Annals Phys. 318, 2 (2005).

[21] B. Abbott et al. (The LIGO Collaboration), Phys. Rev. Lett. 95, 221101 (2005).

[22] T. L. Smith, M. Kamionkowski, and A. Cooray, Phys. Rev. D 73, 023504 (2006). 